\title{
Molecular Origin and Dynamic Behavior of Slip in Sheared Polymer Films
}

\author{
Nikolai V. Priezjev and Sandra M. Troian \\ Department of Chemical Engineering, Princeton University, Princeton, New Jersey 08544, USA
}

(Received 21 April 2003; published 7 January 2004)

\begin{abstract}
The behavior of the slip length in thin polymer films subject to planar shear is investigated using molecular dynamics simulations. At low shear rates, the slip length extracted from the velocity profiles correlates well with that computed from a Green-Kubo analysis. Beyond chain lengths of about $N=10$, the molecular weight dependence of the slip length is dominated strongly by the bulk viscosity. The dynamical response of the slip length with increasing shear rate is well captured by a power law up to a critical value where the momentum transfer between wall and fluid reaches its maximum.
\end{abstract}

DOI: 10.1103/PhysRevLett.92.018302

Slippage at liquid-solid, polymer-solid, and polymerpolymer interfaces can strongly influence hydrodynamic behavior in microscale and nanoscale flows. Contributing factors include poor interfacial wettability or weak molecular attraction between phases [1,2], surface roughness [3], high shear rates [4], a reduction in polymer interfacial viscosity [5], and nucleation of nanobubbles at hydrophobic surfaces [6]. Despite the enormous interest in slip behavior, there is yet no consensus on which parameters control the degree of slip in simple fluids and polymeric systems nor how the slip length depends on the local shear rate.

Navier [7] first proposed that the slip velocity $V_{s}$ at a wall-fluid interface varies linearly with the shear rate $\dot{\gamma}$ according to $V_{s}=L_{s}^{o} \dot{\gamma}$. He assumed a constant (shearindependent) slip length $L_{s}^{o}$, defined as the extrapolated distance into the solid phase where the tangential velocity vanishes. More than a century later, Tolstoi [1] used Frenkel's [8] molecular kinetic theory to link $L_{s}^{o}$ to the liquid-solid equilibrium contact angle in vacuo through the activation energy for molecular displacements. This derivation determined that $L_{s}^{o} \sim a\left(\eta / \eta_{\text {wall }}-1\right)$, where $a$ is the center-center distance between adjacent molecules, $\eta$ is the bulk fluid viscosity, and $\eta_{\text {wall }}$ is the reduced viscosity of the first fluid layer near the wall. Since then, a number of molecular dynamics (MD) studies have focused on the functional dependence of the slip length at low shear rates on molecular parameters affecting momentum transfer at a wall-fluid interface [9-16]. Stratification of the fluid layers near the wall plays an especially significant role [10] in determining the degree of slip. Recent equilibrium studies of simple fluids $[17,18]$ have elucidated the dependence of the slip length on the fluid-wall contact density, the interaction energy, the inplane diffusion coefficient, and the structure factor of the first fluid layer.

Even less is known about the dynamic behavior of the slip length with increasing shear rate. MD simulations [4] of simple liquids in Couette flow have shown that the slip length for Newtonian fluids interacting through a Lennard-Jones (LJ) potential depends nonlinearly on
PACS numbers: 83.50.Lh, 83.10.Rs, 83.50.Rp, 83.80.Sg

the shear rate according to $L_{s}=L_{s}^{*}\left(1-\dot{\gamma} / \dot{\gamma}_{c}\right)^{-1 / 2}$. Here $\dot{\gamma}_{c}$ represents the maximum shear rate the fluid can sustain beyond which there is no additional momentum transfer between the wall and fluid molecules. How generic this behavior is and whether there exists a comparable scaling for polymeric fluids remain open questions.

In this Letter we use MD simulations to examine the dependence of the slip length on molecular parameters and shear rate in thin polymer films subject to planar shear. The emphasis on polymeric fluids is timely since most experiments devoted to slip phenomena rely on polymers in order to diminish evaporative effects and to enhance the degree of slip for measurement purposes [19-22]. Results of our simulations support the view that the low [17] and high shear [4] behavior of the slip length reported earlier for simple fluid systems is more generally applicable to polymeric systems. It is also shown that beyond chain lengths of about $N=10$, the net molecular weight dependence of the slip length at low shear rates is dominated by the bulk fluid viscosity.

The simulation cell consisted of a simple or polymeric fluid (3456 monomers) subject to planar shear in the $\hat{x}$ direction. The fluid monomers interacted through the $\mathrm{LJ}$ potential $V_{\mathrm{LJ}}(r)=4 \epsilon\left[\left(\frac{\sigma}{r}\right)^{12}-\left(\frac{\sigma}{r}\right)^{6}\right]$ with a cutoff distance $r_{c}=2.5 \sigma$, where $\epsilon$ and $\sigma$ represent the energy and length scales of the fluid phase. The wall-fluid parameters were chosen to be $\epsilon_{\mathrm{wf}}=0.6 \epsilon, \sigma_{\mathrm{wf}}=0.75 \sigma$, and $r_{c}=2.5 \sigma$; the fluid phase density $\rho$ and temperature $T$ were held fixed at $0.81 \sigma^{-3}$ and $1.1 \epsilon / k_{B}$, respectively. The polymer fluid was modeled as a collection of bead-spring units $(N=2-16$ beads) interacting through a finitely extensible nonlinear elastic (FENE) potential [23] $V_{\mathrm{FENE}}(r)=$ $-\frac{k}{2} r_{0}^{2} \ln \left[1-r^{2} / r_{0}^{2}\right]$ with $k=30 \epsilon \sigma^{-2}$ and $r_{0}=1.5 \sigma$ [24]. This fluid represents a semidilute polymer melt far below the entanglement regime.

The upper and lower walls of the shear cell each consisted of 1152 atoms distributed between two (111) planes of an fcc lattice of density $\rho_{w}=4 \rho$. The direction of

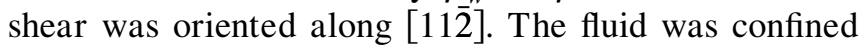
to a gap width $h=24.57 \sigma$ and the entire cell $(x y z)$ 


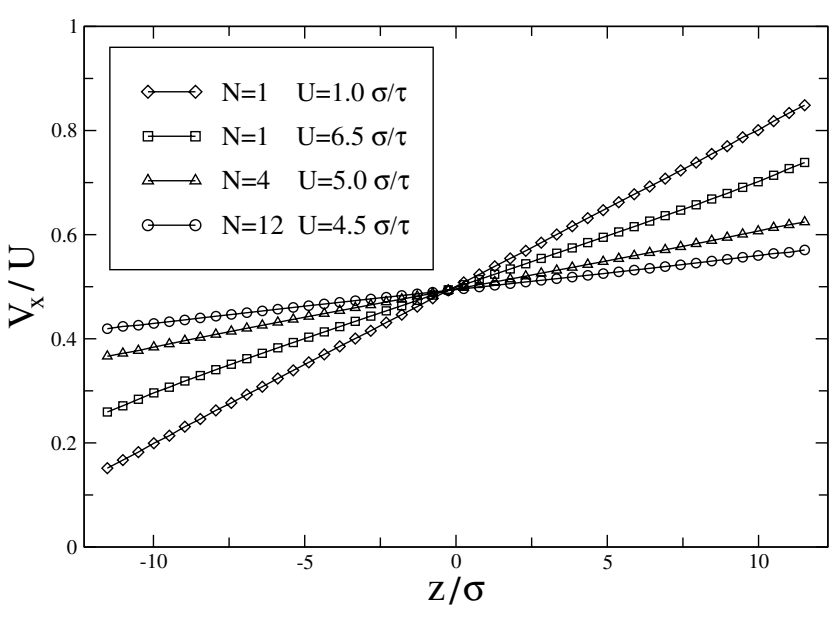

FIG. 1. Sample velocity profiles, $V_{x} / U$, within the shear cell. Slip lengths were estimated from the relation $L_{s}=$ $(U / \dot{\gamma}-h) / 2$ where the shear rate $\dot{\gamma}=d V_{x} / d z$.

measured $25.03 \sigma \times 7.22 \sigma \times h$. Periodic boundary conditions were enforced in the $\hat{x}$ and $\hat{y}$ directions. Constant temperature of the fluid phase was maintained by a Langevin thermostat with friction coefficient $\tau^{-1}$ attached to the $\hat{y}$ degree of freedom for each monomer $[10,25]$. The equations of motion were integrated using a fifth-order Gear predictor-corrector scheme [26] with a time step $\Delta t=0.002 \tau$, where $\tau=\sqrt{m \sigma^{2} / \epsilon}$.

The fluid was sheared by translating the upper wall at a constant speed $U$ while the lower wall remained stationary. After an equilibration period exceeding $10^{5} \tau$, the velocity profile within the fluid, $V_{x}(z / \sigma) / U$, was obtained by averaging the instantaneous monomer speeds in bin widths of $0.5 \sigma$ ( $\hat{z}$ direction) for a period of about $4 \times 10^{5} \tau$. The sample velocity profiles shown in Fig. 1 correspond to the maximum wall velocity imposed for various chain lengths $N$. The velocity fields for $N>1$ remained linear at all shear rates examined. A slight curvature in the velocity profile near the walls was noted for simple fluids $(N=1)$ at wall speeds exceeding $U \geq$ $4.5 \sigma / \tau$ (or equivalently for shear rates exceeding $\dot{\gamma} \geq$ $\left.0.1 \tau^{-1}\right)$. This curvature introduced a maximum error of $0.5 \sigma$ in estimating the slip length. An example of the linear velocity profile for simple fluids $(N=1)$ is also shown in Fig. 1 for $U=1.0 \sigma / \tau$. In this study, the maximum slip velocity at the interface was about $2 \sigma / \tau$. The Reynolds number, based on the maximum fluid velocity and gap height, was of order 10 indicating laminar flow conditions even at the highest shear rates.

Figure 2 shows the viscous response of sheared films with increasing shear rate and chain length (inset). The normalized shear viscosity, $\eta / \epsilon \tau \sigma^{-3}$, was computed from the Kirkwood relation [23]. The maximum shear rate the fluid can sustain before completely slipping decreases with increasing $N$ since longer chain lengths undergo more slippage. Chain lengths below $N=6$ behave as Newtonian liquids throughout; chain lengths

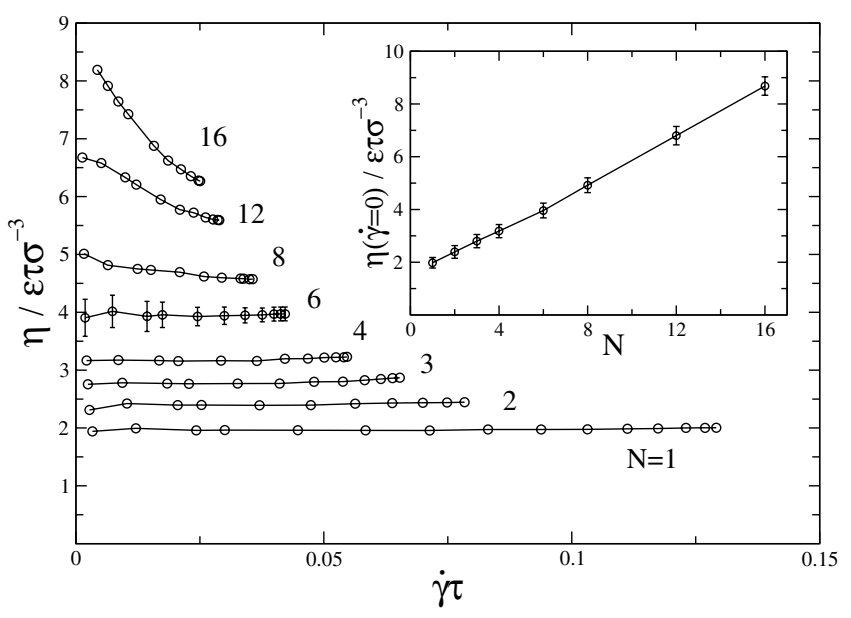

FIG. 2. Behavior of the shear viscosity, $\eta / \epsilon \tau \sigma^{-3}$, as a function of increasing shear rate for chain lengths ranging from $N=1-16$. Inset: dependence of the bulk viscosity in the limit of low shear rates as a function of $N$.

$N \geq 8$ undergo shear thinning. The inset in Fig. 2 shows a monotonic increase in the zero shear viscosity, $\eta(\dot{\gamma} \rightarrow 0)$, with increasing chain length [23].

The dynamic response of the slip length with increasing shear rate for $N$-mers ranging from $N=1-16$ is shown in Fig. 3. The slip length, which is nearly constant at low shear rates, exhibits a strong, nonlinear increase with $\dot{\gamma}$ for both Newtonian and shear thinning fluids. Beyond a critical shear rate the wall can no longer impart additional momentum to the fluid layer. The inset demonstrates that the asymptotic value of the slip length, $L_{s}^{o}$ (obtained by extrapolating the leftmost three points to zero shear rate), increases monotonically with increasing chain length.

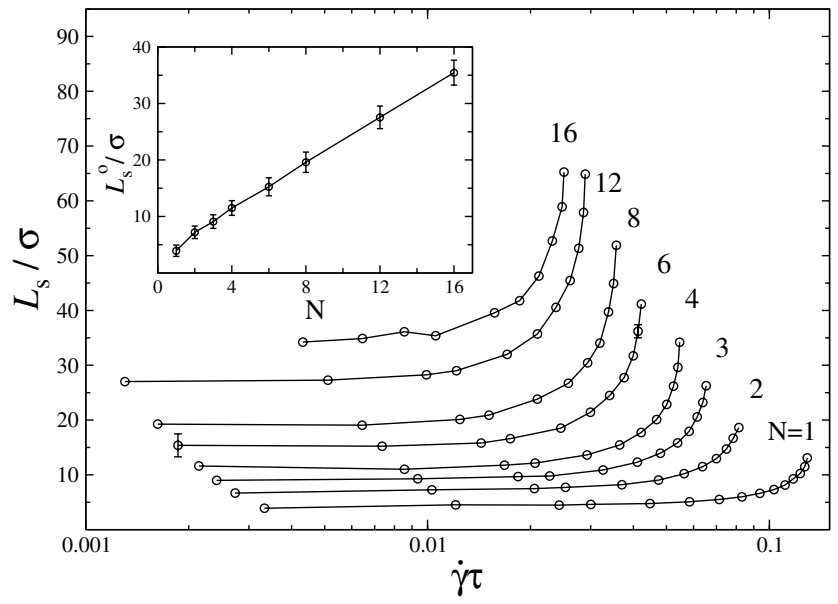

FIG. 3. Behavior of the slip length with increasing shear rate for chain lengths ranging from $N=1-16$. As shown for $N=6$, the errors bars are larger at the lower shear rates because of thermal fluctuations. Inset: variation of the slip length, $L_{s}^{o}$, as a function of chain length $N$ in the limit of low shear rates. 
The data in Fig. 3 can be made to collapse onto a common curve $L_{s} / L_{s}^{*}=\left(1-\dot{\gamma} / \dot{\gamma}_{c}\right)^{-1 / 2}$, as shown in Fig. 4. The values of $L_{s}^{*}(N)$ and $\dot{\gamma}_{c}(N)$, which are tabulated in the inset legend, were obtained by fitting a straight line to the data $L_{s}^{-2}$ versus $\dot{\gamma}$. The vertical and horizontal intercepts of the straight line identify the values $L_{s}^{*}$ and $\dot{\gamma}_{c}$ [27]. The auxiliary slip lengths, $L_{s}^{*}$, were slightly smaller than the extrapolated values $L_{s}^{o}$ in Fig. 3, defined by the low shear regime. This discrepancy indicates that the dynamic collapse holds at the shear rates exceeding $\dot{\gamma} \gtrsim 5 \times 10^{-3} \tau^{-1}$. All cases examined demonstrated a sharp increase in the slip length as $\dot{\gamma} / \dot{\gamma}_{c} \rightarrow 1$. This critical behavior suggests that the slip condition at the wall-fluid interface can affect flow at large distances from the wall.

In addition to the dynamic response, it is interesting to probe exactly what molecular parameters control the degree of slip as $\dot{\gamma} \rightarrow 0$. Barrat and Bocquet investigated this issue [17] for simple fluids $(N=1)$ by appealing to the fluctuation-dissipation theorem. They developed a Green-Kubo analysis for computing the friction coefficient of the first fluid layer near the wall and showed that the slip length at low shear rates, $\delta$, scales according to $\delta \sim \eta D_{q_{\|}} / S\left(q_{\|}\right) \rho_{c}$ for constant temperature and attractive wall-fluid interactions. The wave number, $q_{\|}$, represents the first reciprocal lattice vector of the wall in the direction of shear. $D_{q_{\|}}$, the collective diffusion coefficient, $S\left(q_{\|}\right)$, the structure factor, and, $\rho_{c}$, the contact density, are all quantities computed within the first fluid layer.

We computed by MD simulations the analogous parameters for polymeric fluids. The location of the first fluid layer was estimated from the first minimum in the monomer density profile in the direction perpendicular to

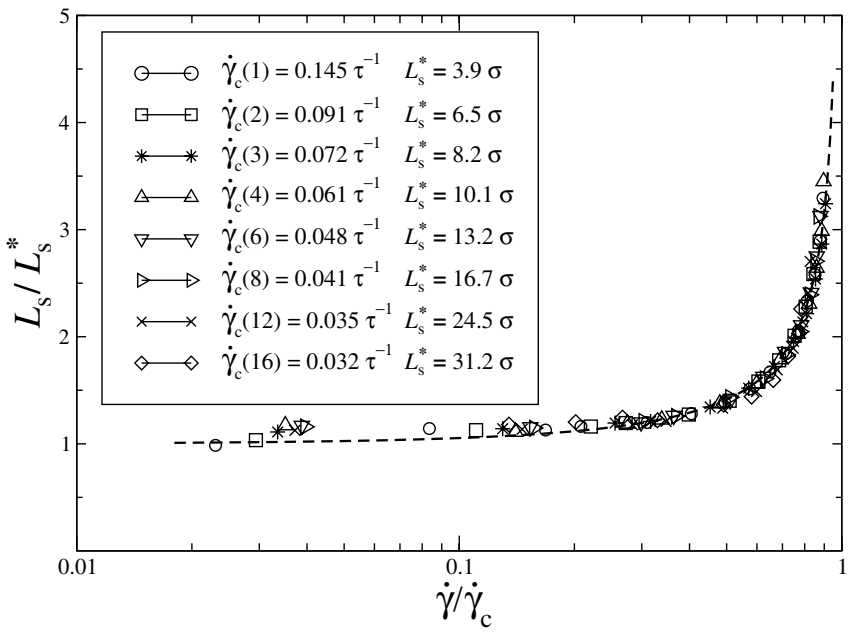

FIG. 4. Master curve describing the dynamic behavior of the slip length for fluid chain lengths ranging from $N=1-16$. The dashed line represents the function $L_{s} / L_{s}^{*}=\left(1-\dot{\gamma} / \dot{\gamma}_{c}\right)^{-1 / 2}$. The legend lists the fitted values of $L_{s}^{*}(N)$ and $\dot{\gamma}_{c}(N)$ used to normalize the data. the wall. The value of the first peak in the profile defined the contact density, $\rho_{c}$. The results show that $\rho_{c}$ decreases with increasing $N$ by about $10 \%$. In our simulations, the reciprocal lattice vector was chosen to be $q_{\|}=6.024 \sigma^{-1}$, which probes distances of about a molecular size. The in-plane structure factor, $S\left(q_{\|}\right)=$ $\left|\sum_{1}^{N_{\ell}} e^{i q_{\|} x}\right|^{2} / N_{\ell}$, where $N_{\ell}$ is the total number of monomers in the first fluid layer, decreases by about $30 \%$ from $N=1$ to 16 . This behavior is expected since polymer chains cannot pack as densely or as orderly as simple fluids near a wall. Computation of the collective diffusion coefficient, $D_{q_{\|}}$, which for polymers reflects internal rearrangements of the chains (and not the $N$-mer center of mass diffusion coefficient), requires an estimate of the decay rate of the density-density autocorrelation function. The data were fit by a Kohlrausch-Williams-Watts stretched exponential according to $\left\langle\rho_{q_{\|}}(t) \rho_{-q_{\|}}(0)\right\rangle=$ $\exp \left(-A q_{\|}^{2} t\right)^{\beta}\left\langle\rho_{q_{\|}}(0) \rho_{-q_{\|}}(0)\right\rangle$, where $A$ and $\beta$ are fitting parameters and $\rho_{q_{\|}}=\sum_{1}^{N_{\ell}} e^{i q_{\|} x}$. The parameter $\beta(N)$ varied from $0.89 \pm 0.03$ for $N=1$ to $0.81 \pm 0.03$ for $N=16$. The slip length, $\delta$, as computed from the expression for the friction coefficient (see Ref. [17]), is proportional to $\int_{0}^{\infty} d t\left\langle\rho_{q_{\|}}(t) \rho_{-q_{\|}}(0)\right\rangle$, from which the collective diffusion coefficient is calculated to be $D_{q_{\|}}=$ $A \beta / \Gamma\left(\beta^{-1}\right)$, where $\Gamma$ is the gamma function.

Each of the separate variables $D_{q_{\|}}, 1 / S\left(q_{\|}\right)$, and $1 / \rho_{c}$ exhibit a similar dependence on $N$ characterized by a sharp increase at small $N$ that asymptotes to a constant value at large $N$ (not shown). Figure 5 shows the behavior of the combined ratio, $D_{q_{\|}} / S\left(q_{\|}\right) \rho_{c}$, normalized by its value for $N=1$. This result indicates that the slip length $\delta(N)$ is dominated by the bulk viscosity above chain lengths of about $N=10$. Tolstoi's model for simple fluids $(N=1)$ also predicts that the slip length at low shear rates is linearly proportional to the bulk viscosity. The

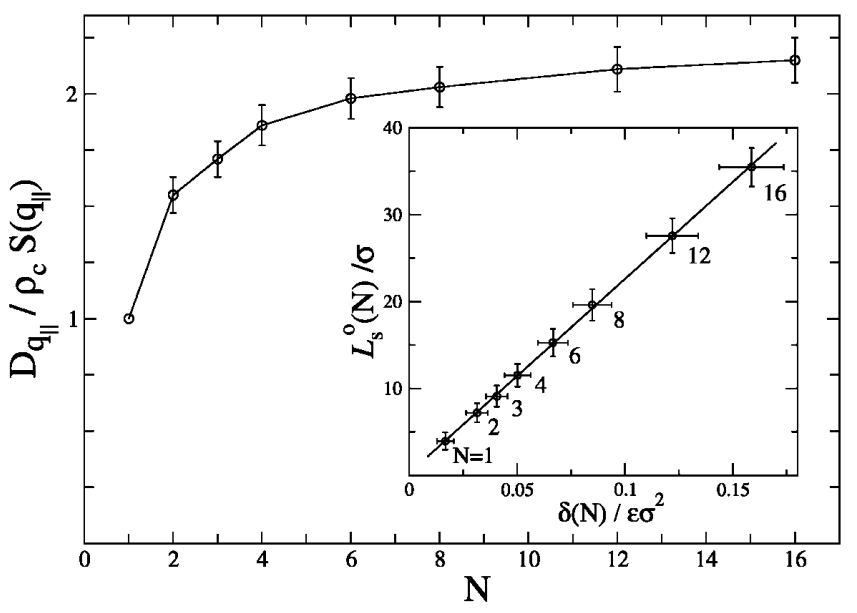

FIG. 5. The dependence of the ratio, $D_{q_{\|}} / S\left(q_{\|}\right) \rho_{c}$ (normalized by its value for $N=1$ ), on chain length $N$. Inset: The correlation between the slip lengths obtained from the shear flow profiles, $L_{s}^{o}(N)$, versus the equilibrium measurements of $\delta(N)$. The solid line $y=223.5 x+0.24$ is plotted for reference. 
inset in Fig. 5 shows a strong correlation between $L_{s}^{o}$ (inset of Fig. 3) and predictions of the slip length evaluated from $\delta \sim \eta D_{q_{\|}} / S\left(q_{\|}\right) \rho_{c}$. This indicates that the Barrat-Bocquet analysis accurately predicts the dependence of the slip length on the parameters described even for polymeric chains that behave as shear thinning fluids.

In summary, we have conducted an MD study of the slip response of $N$-mer polymer chains $(1 \leq N \leq 16)$ subject to planar shear. The first part of the study establishes that the dynamic behavior for the slip length versus shear rate is well described by the function $L_{s} / L_{s}^{*}=(1-$ $\left.\dot{\gamma} / \dot{\gamma}_{c}\right)^{-1 / 2}$. The dynamic collapse holds at shear rates exceeding $\dot{\gamma} \geq 5 \times 10^{-3} \tau^{-1}$. The second part of the study demonstrates that there is a strong correlation between the slip length at low shear rates, obtained from the actual velocity profiles and that predicted from a GreenKubo analysis of the friction coefficient between the wall and the first fluid layer. The simulations also indicate that beyond chain lengths of about $N=10$, the molecular weight dependence of the slip length is mostly dominated by the bulk fluid viscosity. This linear proportionality lends support to Tolstoi's early slip model based on Eyring dynamics.

Further studies will determine how general are these findings. In particular, how sensitive is the slip exponent, $1 / 2$, to the chain length and MD parameters used in this study and how robust are these results to higher molecular weight films subject to shear? The fact that the dynamic behavior of the slip length is captured by a simple functional form for both simple and polymeric fluids suggests the possibility of universal scaling for the slip velocity $V_{s}=L_{s}(\dot{\gamma}) \dot{\gamma}$.

The authors thank Dr. P. A. Thompson of PeerMedia, Inc. for kindly sharing his source code and for helpful discussions. The authors also thank Professor Roberto Car for computational support and useful conversations. Financial support by the National Science Foundation (CTS and DMR divisions), by Unilever Research U.S., by the U.S. Army (TACOM, ARDEC), and NASA is gratefully acknowledged.

[1] D. M. Tolstoi, Dokl. Akad. Nauk SSSR 85, 1089 (1952).

[2] T. D. Blake, Colloids Surf. 47, 135 (1990).

[3] S. Richardson, J. Fluid Mech. 59, 707 (1973).

[4] P. A. Thompson and S. M. Troian, Nature (London) 389, 360 (1997).

[5] F. Brochard, P. G. DeGennes, and S. M. Troian, C. R. Acad. Sci., Ser. II 310, 1169 (1990).
[6] O. I. Vinogradova, N. F. Bunkin, N.V. Churaev, O. A. Kiseleva, A.V. Lobeyev, and B.W. Ninham, J. Colloid Interface Sci. 173, 443 (1995).

[7] C. L. M. H. Navier, Mem. Acad. Sci. Inst. Fr. 6, 839 (1827).

[8] J. I. Frenkel, Theory of Liquids (Oxford University Press, Oxford, 1946).

[9] I. Bitsanis, J. J. Magda, M. Tirrell, and H.T. Davis, J. Chem. Phys. 87, 1733 (1987).

[10] P. A. Thompson and M. O. Robbins, Phys. Rev. A 41, 6830 (1990).

[11] P. A. Thompson, G. S. Grest, and M. O. Robbins, Phys. Rev. Lett. 68, 3448 (1992).

[12] R. Khare, J. J. de Pablo and A. Yethiraj, Macromolecules 29, 7910 (1996).

[13] P. A. Thompson, M. O. Robbins, and G. S. Grest, Isr. J. Chem. 35, 93 (1995).

[14] M. J. Stevens, M. Mondello, G. Crest, S. T. Cui, H. D. Cochran, and P.T. Cummings, J. Chem. Phys. 106, 7303 (1997).

[15] E. Manias, G. Hadziioannou, I. Bitsanis, and G. ten Brinke, Europhys. Lett. 24, 99 (1993).

[16] A. Jabbarzadeh, J. D. Atkinson, and R. I. Tanner, J. Chem. Phys. 110, 2612 (1999).

[17] J.-L. Barrat and L. Bocquet, Phys. Rev. Lett. 82, 4671 (1999); Faraday Discuss. 112, 119 (1999).

[18] L. Bocquet and J.-L. Barrat, Phys. Rev. E 49, 3079 (1994).

[19] R. G. Larsen, The Structure and Rheology of Complex Fluids (Oxford University Press, New York, 1999), Chap. 1 and references therein.

[20] Y. Zhu and S. Granick, Phys. Rev. Lett. 87, 096105 (2001).

[21] K. B. Migler, H. Hervet, and L. Leger, Phys. Rev. Lett. 70, 287 (1993).

[22] R. G. Horn, O. I. Vinogradova, M. E. Mackay, and N. Phan-Thien, J. Chem. Phys. 112, 6424 (2000).

[23] R. B. Bird, C. F. Curtiss, R. C. Armstrong, and O. Hassager Dynamics of Polymeric Liquids (Wiley, New York, 1987), 2nd ed.

[24] K. Kremer and G. S. Grest, J. Chem. Phys. 92, 5057 (1990).

[25] G. S. Grest and K. Kremer, Phys. Rev. A 33, 3628 (1986).

[26] M. P. Allen and D. J. Tildesley, Computer Simulation of Liquids (Clarendon, Oxford, 1987).

[27] Fluids consisting of longer chains showed a slight downward curvature at the low shear rates when plotted as $L_{s}^{-2}$ versus $\dot{\gamma}$. The deviation from linearity may be due to finite size effects since fluids consisting of longer chains produced slip lengths exceeding the cell gap width $h$. Adjustment of the inverse exponent of $L_{s}$ (the ordinate axis) to produce the best line fit yielded a slip exponent variation of $0.5 \pm 0.05$. 